Fixed Point Theory, 20(2019), No. 2, 649-662

DOI: $10.24193 /$ fpt-ro.2019.2.43

http://www.math.ubbcluj.ro/ nodeacj/sfptcj.html

\title{
COMMON FIXED POINT THEOREMS IN C*-ALGEBRA-VALUED B-METRIC SPACES WITH APPLICATIONS TO INTEGRAL EQUATIONS
}

\author{
S.S. RAZAVI* AND H.P. MASIHA**,1 \\ *Faculty of Mathematics, K. N. Toosi University of Technology \\ Tehran, Iran \\ E-mail: srazavi@mail.kntu.ac.ir \\ **Faculty of Mathematics, K. N. Toosi University of Technology \\ P.O. Box 16315-1618, Tehran, Iran \\ E-mail: masiha@kntu.ac.ir
}

\begin{abstract}
Based on the concepts of $\mathrm{C}^{*}$-algebra-valued b-metric space, we give some common fixed point results in $\mathrm{C}^{*}$-algebra-valued b-metric space. As an application, existence and uniqueness result for one type of integral equations is also discussed.

Key Words and Phrases: Fixed point, b-metric space, $\mathrm{C}^{*}$-algebra, common fixed point, compat-
\end{abstract} ible, weakly compatible.

2010 Mathematics Subject Classification: 47H10, 54H25.

\section{REFERENCES}

[1] M. Abbas, G. Jungck, Common fixed point results for noncommuting mappings without continuity in cone metric spaces, J. Math. Anal. Appl., 341(2008), 416-420.

[2] M.T. Abu Osman, Fuzzy metric space and fixed fuzzy set theorem, Bull. Malaysian Math. Soc., 6(2)(1983), 1-4.

[3] A. Amini-Harandi, H. Emami, A fixed point theorem for contraction type maps in partially ordered metric spaces and application to ordinary differential equations, Nonlinear Anal., 72(5)(2010), 2238-2242.

[4] I.A. Bakhtin, The contraction mapping principle in almost metric spaces, Funct. Anal., 30(1989), 26-37.

[5] V. Berinde, A common fixed point theorem for compatible quasi contractive self mappings in metric spaces, Appl. Math. Comput., 213(2009), no. 2, 348-354.

[6] M. Boriceanu, M. Bota, A. Petruşel, Mutivalued fractals in b-metric spaces, Central Eur. J. Math., 8(2010), no. 2, 367-377.

[7] B.S. Choudhury, N. Metiya, The point of coincidence and common fixed point for a pair of mappings in cone metric spaces, Appl. Math. Comput., 60(2010), no. 6, 1686-1695.

[8] L.B. Ćirić, B. Samet, H. Aydi, C. Vetro, Common fixed points of generalized contractions on partial metric spaces and an application, Appl. Math. Comput., 218(2011), no. 6, 2398-2406.

[9] G. Cortelazzo, G. Milan, G. Vezzi, P. Zamoeroni, Trademark shapes description by string matching techniques, Pattern Recognit., 27(1994), no. 8, 1005-1018.

${ }^{1}$ Corresponding author. 
[10] S. Czerwik, Contraction mappings in b-metric spaces, Acta Math. Inform. Univ. Ostrava, 1(1993), 5-11.

[11] S. Czerwik, Nonlinear set-valued contraction mappings in b-metric spaces, Atti Sem. Mat. Fis. Univ. Modena, 46(1998), no. 2, 263-276.

[12] R.G. Douglas, Banach Algebra Techniques in Operator Theory, Acad. Press, 46(1972).

[13] J. Esmaily, S.M. Vaezpour, B.E. Rhoades, Coincidence point theorem for generalized weakly contractions in ordered metric spaces, Appl. Math. Comput., 219(2012), no. 4, 1536-1548.

[14] R. Fagin, L. Stockmeyer, Relaxing the triangle inequality in pattern matching, Int. J. Comput. Vis., 30(1998), no. 3, 219-231.

[15] J. Harjani, K. Sadarangani, Generalized contractions in partially ordered metric spaces and applications to ordinary differential equations, Nonlinear Anal., 72(2010), 1188-1197.

[16] L. Huang, X. Zhang, Cone metric spaces and fixed point theorems of contractive mappings, J. Math. Anal. Appl., 332(2007), 1468-1476.

[17] N. Hussian, M.H. Shah, KKM mappings in cone b-metric spaces, Comput. Math. Appl., 62(2011), 1677-1684.

[18] S. Janković, Z. Golubović, S. Radenović, Compatible and weakly compatible mappings in cone metric spaces, Math. Comput. Model., 52(2010), 1728-1738.

[19] G. Jungck, Commuting mappings and common fixed points, Amer. Math. Monthly, 73(1966), 735-738.

[20] G. Jungck, Compatible mappings and common fixed points, Int. J. Math. Math. Sci., 9(1986), 771-779.

[21] G. Jungck, S. Radenović, S. Radojević, V. Rakocević, Common fixed point theorems for weakly compatible pairs on cone metric spaces, Fixed Point Theory Appl., (2009), Article ID 643840

[22] W. Kirk, N. Shahzad, Fixed Point Theory in Distance Spaces, vol. XI, Springer, 2014, p. 173.

[23] Z.H. Ma, L.N. Jiang, $C^{*}$-algebra-valued b-metric spaces and related fixed point theorems, Fixed Point Theory Appl., (2015).

[24] Z.H. Ma, L.N. Jiang, Q.L. Xin, Fixed point theorems on operator-valued metric space, Trans. Beijing Inst. Tech., 34(10)(2014), 1078-1080.

[25] Z.H. Ma, L.N. Jiang, H.K. Sun, $C^{*}$-algebra-valued metric spaces and related fixed point theorems, Fixed Point Theory Appl., 2014(2014), Art. ID 206.

[26] R. McConnell, R. Kwok, J. Curlander, W. Kober, S. Pang, $\Psi-s$ Correlation and dynamic time warping: two methods for tracking ice floes, IEEE Trans. Geosci. Remote Sens., 29(1991), no. 6, 1004-1012.

[27] G.J. Murphy, C*-Algebras and Operator Theory, Academic Press, London, 1990.

[28] W. Shatanawi, M. Postolache, Common fixed point theorems for dominating and weak annihilator mappings in ordered metric spaces, Fixed Point Theory Appl., (2013), Art. ID 271.

[29] L. Shi, S. Xu, Common fixed point theorems for two weakly compatible self-mappings in cone b-metric spaces, Fixed Point Theory Appl., (2013), Art. ID 120.

[30] E. Tarafdar, An approach to fixed-point theorems on uniform spaces, Trans. Amer. Math. Soc., 191(1974), 209-225.

[31] Q. Xia, The geodesic problem in quasimetric spaces, J. Geom. Anal., 19(2009), no. 2, 452-479.

[32] Q.L. Xin, L.N. Jiang, Common fixed point theorems for generalized k-ordered contractions and B-contractions on noncommutative Banach spaces, Fixed Point Theory Appl., (2015), Art. ID 77.

[33] Q.L. Xin, L.N. Jiang, Z.H. Ma, Common fixed point theorems in $C^{*}$-algebra-valued metric spaces, (in progress).

[34] Z.H. Yanga, H. Sadatib, S.H. Sedghib, N. Shobec, Common fixed point theorems for noncompatible self-maps in b-metric spaces, J. Nonlinear Sci. Appl., 8(2015), 1022-1031.

Received: June 7, 2017; Accepted: March 9, 2018. 
FIXED POINT THEOREMS IN C*-ALGEBRA-VALUED B-METRIC SPACES 